BULLETIN OF THE

AMERICAN MATHEMATICAL SOCIETY

Volume 77, Number 6, November 1971

\title{
LOCALIZATION AND COMPLETION IN HOMOTOPY THEORY ${ }^{1}$
}

\author{
BY A. K. BOUSFIELD AND D. M. KAN
}

Communicated by E. H. Brown, June 1, 1971

1. Introduction. For each space $X$ (i.e. simplicial set with only one vertex) and solid ring $R$ (i.e. commutative ring with 1 , for which the multiplication map $R \otimes R \rightarrow R$ is an isomorphism [3]) we shall construct, in a functorial manner, a space $X_{R}$, the $R$-completion of $X$, and discuss some of its properties. The proofs will be given elsewhere.

If $R \subset Q$ (i.e. $R$ is a subring of the rationals) and $\pi_{1} X=0$, then $\pi_{*} X_{\hat{R}} \approx \pi_{*} X \otimes R$ and $X_{R}^{\hat{R}}$ is a localization in the sense of [7], [9] and [11].

If $R=Z_{p}$ (the integers modulo a prime $p$ ), $\pi_{1} X=0$ and $\pi_{n} X$ is finitely generated for all $n$, then $\pi_{*} X_{R}$ is the usual $p$-profinite completion of $\pi_{*} X$ and $X_{R}$ is a p-completion in the sense of [8] and [11].

This note is, in some sense, a continuation of [2]. However, our present construction is (although homotopically equivalent to) completely different from the one of [2] and has the advantage that it can easily be generalized to a functorial notion of fibre-wise R-completion. In [2] we used cosimplicial methods, while here the basic tool is that of

2. The $R$-completion of a group. To define this notion we call a group $N$ an $R$-nilpotent group if $N$ has a central series

$$
1=N_{k} \subset \cdots \subset N_{j} \subset \cdots \subset N_{0}=N
$$

such that for each $j$ the quotient group $N_{j} / N_{j+1}$ admits an $R$-module structure. The $R$-completion of a group $G$ then is the group $G_{R}^{\hat{R}}$ obtained by combining Artin-Mazur $[1, \S 3]$ with an inverse limit, i.e. by taking the inverse limit $[1$, p. 147] of the functor which assigns to every homomorphism $G \rightarrow N$, where $N$ is $R$-nilpotent, the group $N$, and to every commutative triangle<smiles></smiles>

AMS 1969 subject classifications. Primary 5540.

$K e y$ words and phases. Relative $R$-completion of groups, $R$-completion of spaces, fibre-wise $R$-completion, $R$-nilpotent groups, $R$-nilpotent spaces.

1 This research was supported by the National Science Foundation. 
with $N$ and $N^{\prime}$ both $R$-nilpotent, the map $N \rightarrow N^{\prime}$. Clearly this $R$-completion comes with a natural map $G \rightarrow \hat{G_{R}}$ and indeed the functor ()$_{\hat{R}}$ has the structure of a triple on the category of groups.

Some "well-known" special cases are:

I. If $R=Z$ (the integers), then $G_{R}=\operatorname{proj} \lim G / \Gamma_{i} G$, where $\left\{\Gamma_{i} G\right\}$ is the lower central series [4].

II. If $R=Z_{p}$, then $G_{R}^{\hat{n}}=\operatorname{proj} \lim G / \Gamma_{i}^{(p)} G$, where $\left\{\Gamma_{i}^{(p)} G\right\}$ is the p-lower central series [4].

III. If $R=Z_{p}$ and $G$ is finitely generated, then $G_{R}$ is the p-profinite completion of Serre [10, I-5] and thus if $G$ is also abelian, then $G_{R} \approx G \otimes$ (the $p$-adic integers).

IV. If $R=Q$ and $G$ is nilpotent (i.e. $Z$-nilpotent), then $G_{\hat{R}}$ is the Mal'cev completion [5], [9, p. 279].

V. If $\mathrm{R} \subset Q$ and $G$ is abelian, then $G_{R} \approx G \otimes R$.

It is not hard to verify that there also is a notion of relative $R$-completion, which assigns to a short exact sequence of groups $1 \rightarrow F \rightarrow G$ $\rightarrow H \rightarrow 1$ a commutative diagram

$$
\begin{aligned}
& 1 \rightarrow F \rightarrow G \rightarrow H \rightarrow 1
\end{aligned}
$$

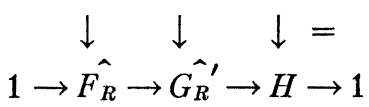

with the bottom row also exact. Here $G_{R}{ }^{\prime}$ is the group with generators $(g, h)$, where $g \in F_{R}$, and relations

$$
\begin{aligned}
(g f, h) & =(g, f h), \quad f \in F, g \in G, h \in \hat{F_{R}}, \\
(g, h)\left(g^{\prime}, h^{\prime}\right) & =\left(g g^{\prime}, g^{\prime}(h) h^{\prime}\right), \quad g, g^{\prime} \in G, h, h^{\prime} \in \hat{F_{R},}
\end{aligned}
$$

where $g^{\prime}$ also denotes the automorphism of $F_{R}$, which is the $R$-completion of the automorphism of $F$, which, in turn, is the restriction of the inner automorphism of $G$ induced by $g^{\prime}$.

3. The $R$-completion of a space. Let $X$ be a "space," i.e. a simplicial set with only one vertex. Then we define its $R$-completion, $X_{R}^{\hat{R}}$, by

$$
\hat{X_{R}}=\bar{W}(G X) \hat{R}
$$

i.e. we apply to $X$ the loop group functor $G$ [6], then $R$-complete dimensionwise and finally apply the classifying functor $W$ [6]. Clearly this $R$-completion also comes with a natural map

$$
X \rightarrow \bar{W} G X \rightarrow \bar{W}(G X) \hat{R_{R}}=\hat{X \hat{R}}
$$

and this functor ()$_{\hat{R}}$ has the structure of a triple on the category of "spaces." 
Using the relative $R$-completion one can, in the same way, obtain the fibrewise $R$-completion of a fibration of "spaces" $F \rightarrow E \rightarrow B$, i.e. a functorial commutative diagram

$$
\begin{aligned}
& F \rightarrow E \rightarrow B \\
& \downarrow \quad \downarrow \quad \downarrow= \\
& \hat{F_{R}} \rightarrow \hat{E_{R}^{\prime}} \rightarrow B
\end{aligned}
$$

for which the map $E_{\hat{R}^{\prime}} \rightarrow B$ is a fibre map and the inclusion of $F_{R}$ in its fibre is a homotopy equivalence.

Depending on how much $X_{R}^{\hat{R}}$ resembles $X$, one can consider three classes of spaces: $R$-complete, $R$-good and $R$-bad ones. First the

4. $R$-complete spaces. A space $X$ will be called $R$-complete if the natural map $X \rightarrow X_{R}^{\hat{R}}$ induces an isomorphism $\pi_{*} X \approx \pi_{*} X_{R}^{\hat{R}}$. For example

4.1 The space $K(B, n)$ is $R$-complete if $B$ admits an $R$-module structure $(n \geqq 1)$.

To obtain a wider class of $R$-complete spaces we will say that a group $\pi$ acts $R$-nilpotently on a left $\pi$-module $M$, if $M$ has a $\pi$-module filtration

$$
1=M_{k} \subset \cdots \subset M_{j} \subset \cdots \subset M_{0}=M
$$

such that each quotient $M_{j} / M_{j+1}$ has trivial $\pi$-action and admits an $R$-module structure, and call a space $X R$-nilpotent if $\pi_{1} X$ is $R$-nilpotent and $\pi_{1} X$ acts $R$-nilpotently on $\pi_{n} X$ for $n \geqq 2$. This is equivalent to saying that the Postnikov tower of $X$ can be refined to a tower of principal fibrations where each fibre is a $K(B, n)$ with $B$ an $R$-module. Now we have

\subsection{Every $R$-nilpotent space is $R$-complete.}

This follows readily from 4.1 and the following lemma (with $Y=*$ ).

4.3 Fibre Square Lemma. Consider the two commutative squares of connected spaces

$$
\begin{aligned}
& F \rightarrow X \\
& \downarrow \\
& Y \rightarrow B
\end{aligned}
$$$$
\begin{aligned}
& \hat{F_{R}} \rightarrow \hat{X_{R}} \\
& \downarrow \\
& \hat{Y_{R}} \rightarrow \hat{B_{R}}
\end{aligned}
$$

If the first one of these is a fibre square with simply connected lower right corner, then so is, up to homotopy, the second one. 
5. $R$-good spaces. A space $X$ will be called $R$-good if the natural map $X \rightarrow X_{R}^{\hat{R}}$ induces an isomorphism $H_{*}(X ; R) \approx H_{*}\left(X_{R}^{\hat{R}} ; R\right)$. Moreover one has

$5.1 A$ space $X$ is $R$-good if and only if $X_{R}^{\hat{R}}$ is $R$-complete.

This follows, using the triple structure of ()$_{R}$, from

$5.2 \mathrm{~A}$ map $f: X \rightarrow Y$ induces an isomorphism $H_{*}(X ; R) \approx H_{*}(Y ; R)$ if and only if it induces an isomorphism $\pi_{*} X_{R} \approx \pi_{*} Y_{R}$.

Another consequence of this is that for an $R$-good space $X$ the natural map $i: X \rightarrow X_{R}$ is, up to homotopy, characterized by each of the following universal properties:

(i) For any map $f: X \rightarrow Y$ inducing an isomorphism $H_{*}(X ; R)$ $\approx H_{*}(Y ; R)$ there is a unique homotopy class of maps $g: Y \rightarrow X_{R}$ such that $g f \sim i$.

(ii) For any map $f: X \rightarrow Y$ where $Y$ is $R$-complete, there is a unique homotopy class of maps $h: X_{R} \rightarrow Y$ such that hi $\sim f$.

It seems hard to say when a space is $R$-good. Some partial results in this direction are

5.3 Let $R=Z_{p}$ or $R \subset Q$ and let $B$ be an abelian group. Then $K(B, n)$ is $R$-good for all $n$.

Combining this with 4.3 one gets

5.4 Let $R=Z_{p}$ or $R \subset Q$. Then every $Z$-nilpotent space is $R$-good.

This is not best possible. For instance one has

5.5 Let $R=Z_{p}$ or $R \subset Q$ and let $X$ be a space such that $H_{1}(X ; R)=0$. Then $X$ is $R$-good.

6. $R$-bad spaces. A space $X$ is called $R$-bad if it is not $R$-good. It turns out that $R$-bad spaces are "very bad."

6.1 If $X$ is $R$-bad, then so is $X_{R}^{\hat{R}}$, i.e. no iterated $R$-completion of $X$ is $R$-complete.

An example of a $Z$-bad space is an infinite wedge of circles, but we do not know whether the wedge of two circles is $Z$-bad.

7. Homotopy groups of an $R$-completion. The following result illustrates the close relation between the $R$-completion functor for spaces and the one for groups.

7.1 Let $X$ be a $Z$-nilpotent space, and unless $R \subset Q$, suppose that $\pi_{n} X$ is finitely generated for all $n$. Then there is a natural isomorphism 


$$
\pi_{n}\left(\hat{X_{R}}\right) \approx\left(\pi_{n} X\right)_{R}, \quad n \geqq 1 .
$$

This is proved by combining 4.3 with computations for $K(B, n)$ 's. For more results in the case $R=Z_{p}$ see $[2,7.2]$.

We end with the following consequence of 4.3 and 7.1 , which is useful in computing $R$-completions of groups:

7.2 Let $N$ be a nilpotent (i.e. Z-nilpotent) group, let $B \subset N$ be a normal subgroup and, unless $R \subset Q$, suppose that $N$ is finitely generated. Then the following sequence is exact:

$$
1 \rightarrow \hat{B_{R}} \rightarrow \hat{N_{R}} \rightarrow\left(N / B \hat{)_{R}} \rightarrow 1\right.
$$

\section{REFERENCES}

1. M. Artin and B. Mazur, Etale homotopy, Lecture Notes in Math., no. 100, Springer-Verlag, Berlin and New York, 1969. MR 39 \#6883.

2. A. Bousfield and D. Kan, Homotopy with respect to a ring, Proc. Sympos. Pure Math., vol. 22, Amer. Math. Soc., Providence, R. I., 1971, pp. 59-64.

3. - The core of a ring (to appear).

4. E. Curtis, Simplicial homotopy theory, Advances in Math. 6 (1971), 107-209.

5. A. Mal'cev, Nilpotent groups without torsion, Izv. Akad. Nauk SSSR Ser. Mat. 13 (1949), 201-212. (Russian) MR 10, 507.

6. J. P. May, Simplicial objects in algebraic topology, Van Nostrand Math. Studies, no. 11, Van Nostrand, Princeton, N. J., 1967. MR 36 \#5942.

7. M. Mimura, G. Nishida and H. Toda, Localization of CW-complexes and its applications (to appear).

8. D. Quillen, An application of simplicial profinite groups, Comment Math. Helv. 44 (1969), 45-60. MR 39 \#3490.

9. - Rational homotopy theory, Ann. of Math. (2) 90 (1969), 205-295. MR 41 \#2678.

10. J.-P. Serre, Cohomologie galoisienne, Lecture Notes in Math., no. 5, SpringerVerlag, Berlin and New York, 1964. MR 31 \#4785.

11. D. Sullivan, Geometric topology. I: Localization, periodicity and Galois symmetry, M.I.T., Cambridge, Mass., 1970 (mimeographed notes).

Brandeis University, Waltham, Massachusetts 02154

Massachusetts Institute of Technology, Cambridge, Massachusetts 02139 